\title{
The last globally stable extended alkane*
}

\author{
Nils O. B. Lüttschwager, Tobias N. Wassermann, \\ Ricardo A. Mata ${ }^{a}$, Martin A. Suhm ${ }^{b}$ \\ Institut für Physikalische Chemie, Universität Göttingen, Tammannstr. 6, 37077 Göttingen, \\ Germany
}

submitted to Angewandte Chemie as a Communication

3 figures and 6 manuscript pages

Mother of All Folding: Cold isolated linear alkanes $\mathrm{C}_{n} \mathrm{H}_{2 n+2}$ prefer an extended all-trans conformation before cohesive forces between the chain ends induce a folded hairpin structure for longer chains. It is shown by Raman spectroscopy at $100-150 \mathrm{~K}$ that the folded structure becomes more stable beyond $n_{c}=17-18$ carbon atoms. High level quantum chemical calculations yield $n_{c}=17 \pm 1$ as the critical chain length.

aE-mail: rmata@gwdg.de

bE-mail: msuhm@gwdg.de

*The present work was supported by the DFG (Su 121/2 and RTG 782). M. Albrecht has contributed to the early design stage of the heated nozzle. R. Balabin was originally assigned to be part of this project during an undergraduate research project in 2007.

Supporting information is available on the WWW under ... 
At low temperatures, linear alkanes $\mathrm{C}_{n} \mathrm{H}_{2 n+2}$ of moderate length are known to prefer a fully extended (all-trans) conformation in analogy to the simplest case of butane. ${ }^{[1]}$ Due to weak dispersion interactions between chain segments, this cannot hold up to $n \rightarrow \infty$. Only a finite, quite moderate ${ }^{[1,2]}$ amount of energy is required to bend an extended chain into a hairpin structure via four trans-gauche isomerizations. With increasing $n$, the self-solvation between the two aligned chain segments grows and will win over the deformation energy at some point. ${ }^{[2]}$ The theoretical minimum structure predicted for a given $n$ will depend sensitively on the details of the alkane model. ${ }^{[2]}$ The maximum chain length $n_{c}$ with an all-trans global minimum is a quality indicator for the balance between intra- and intermolecular forces. One finds $n_{c} \approx 11$ for PM3, 17 for MM2, 21 for OPLS, ${ }^{[3]} 24$ for MM3, 25 for AMBER and 59 for AM1. ${ }^{[2]}$ Recent predictions fall in the range $15-17^{[3]}$ and $15-21 .^{[4]}$ Experimentally, $n_{c}$ remains unknown. Experimental lower and upper bounds for $n_{c}$ are valuable for an assessment of very accurate ab initio and empirically modified $^{[4]}$ quantum chemical descriptions of alkanes. They can be used for the training of molecular dynamics force fields. ${ }^{[5]}$

Any experimental strategy to determine $n_{c}$ must involve cold isolated alkanes in the gas phase. Low temperature is essential, as there is an overwhelming number of non-extended conformations which quickly dominate the isomer distribution for long alkane chains with increasing temperature. Already for pentane at room temperature, the all-trans conformation is no more the dominant one ${ }^{[6,7]}$ Due to the low volatility of long alkanes, a non-equilibrium technique is unavoidable. Supersonic jet expansions generate molecules with rotational and translational temperatures around $10 \mathrm{~K} .{ }^{[8]}$ It is more challenging to lower the vibrational and conformational temperatures via collisions below the point where the global minimum structure starts to dominate. ${ }^{[9]}$ Concerning detection, a characteristic vibration of the all-trans structure in the Raman spectrum ${ }^{[10]}$ stands out among the less specific vibrations of folded alkanes. The frequency of this so-called accordion vibration is roughly inversely proportional to $n$. The chain ends oscillate symmetrically relative to the chain center, ${ }^{[9]}$ maximizing the change in polarizability. At a given temperature, the accordion Raman intensity relative to the conformationally less sensitive integrated $\mathrm{CH}$ stretching manifold should drop as a function of chain length, because more and more single- and multiple-gauche conformations will compete - a conformational entropy effect. An accelerated decrease is expected beyond $n_{c}$, when energetically competing hairpin structures emerge. The conformational distribution in a supersonic jet will deviate from a Boltzmann distribution, because the strongly populated single- 
gauche alkanes are more likely to relax to the all-trans structure than to a hairpin structure requiring three specific trans-gauche isomerizations. Therefore, the disappearance of the all-trans structure only provides a loose upper bound for $n_{c}$.

To obtain a reliable theoretical estimate of $n_{c}$, we have performed a series of first principles calculations for $n=14-22$. The energy difference is rather small in this size range. This requires a high-level treatment, with a balanced description of the torsion potentials as well as the dispersion interactions responsible for the stability of the hairpin. Our approach is based on density fitted local correlation methods. ${ }^{[11-14]}$ This class of methods offers substantial reductions in the computational cost but also involves approximations which should be carefully considered in this context. There are two main sources of error. One is due to a truncation of the virtual space (domain error). This error is reduced when approaching the complete basis set limit. ${ }^{[15]}$ Furthermore, in local coupled cluster treatments, orbital pairs separated by at least one bond are by default treated at the MP2 level (weak pair approximation). This behavior can be controlled by distance criteria. We have performed a careful analysis of these effects (see Supplement) and devised a composite approach to reduce the above mentioned errors.

The cc-pVTZ (VTZ) and aug-cc-pVTZ basis sets (AVTZ) ${ }^{[16,17]}$ were used throughout. The structures of all conformers were optimized at the DF-LMP2/VTZ level of theory. The electronic energy of each structure was then refined by computing:

$$
E=E(\mathrm{DF}-\mathrm{LMP} 2-\mathrm{F} 12 / \mathrm{VTZ}-\mathrm{F} 12)+\Delta E_{s r}(\mathrm{CC} / \mathrm{AVTZ})+\Delta E_{l r}(\mathrm{CC} / \mathrm{VTZ})
$$

where

$$
\begin{gathered}
\Delta E_{s r}(\mathrm{CC} / \mathrm{AVTZ})=E_{s r}(\mathrm{DF}-\mathrm{LCCSD}(\mathrm{T} 0) / \mathrm{AVTZ})-E(\mathrm{DF}-\mathrm{LMP} 2 / \mathrm{AVTZ}) \\
\Delta E_{l r}(\mathrm{CC} / \mathrm{VTZ})=E_{l r}(\mathrm{DF}-\mathrm{LCCSD}(\mathrm{T} 0) / \mathrm{VTZ})-E_{s r}(\mathrm{DF}-\mathrm{LCCSD}(\mathrm{T} 0) / \mathrm{VTZ})
\end{gathered}
$$

The first term in Eq. (1) corresponds to the DF-LMP2-F12/3* $\mathrm{A}^{[18]}$ energy using the VTZ-F12 basis set. ${ }^{[19]}$ Through the use of explicit correlation, we are able to significantly reduce the basis set as well as the domain error in the underlying LMP2 calculation. The two remaining terms include higher-order correlation effects through the use of density fitting CCSD with noniterative perturbative triples [DF-LCCSD(T0)]. In calculations with the $\operatorname{sr}(=$ short-range) subscript, the default pair distances are used. In this way, only short-range contacts are treated at the highest level. Although the largest corrections are found for such pairs, one needs to increase the distance criterion in order to treat interactions between the parallel chains at the coupled cluster level. This 
is done in the calculations with the $\operatorname{lr}(=$ long-range $)$ subscript, where the distance criterion for strong pairs is increased. The scaled zero point vibrational energy DF-LMP2/VTZ values (scaling factor of 0.978$)^{[20]}$ are then added to the electronic energy values obtained with Eq. (1), together with thermal corrections $(100 \mathrm{~K})$. Further details relative to the distance criteria used and the overall computational procedure, together with benchmark calculations for the pentane system, can be found in the Supplement.

The results are shown in Fig. 1. One should notice that the energy difference varies very slowly with increasing size. This means that very small errors in the calculations might shift the energetic crossover point significantly. Taking into account the approach chosen, the largest error should be in determining the zero point corrections. It is difficult to assess the latter accurately, but an estimated uncertainty of \pm 1 carbon atom or about $2 \mathrm{~kJ} / \mathrm{mol}$ in the energy difference appears reasonable. This leads to a prediction of $n_{c}=17 \pm 1$.

To access $n_{c}$ experimentally, supersonic jet expansions of linear alkanes with $n=13-20$ were characterized by Raman spectroscopy. The expansions were probed $1 \mathrm{~mm}$ downstream a $4.0 \mathrm{~mm} \times 0.15 \mathrm{~mm}$ heated slit nozzle, using $0.5-0.9$ bar He as a carrier gas, partially doped with Ar or $\mathrm{CF}_{4}$ to enhance energy transfer. In the range $n=16-20$, the all-trans enhancement amounts to $30 \pm 10 \%$ for $4 \% \mathrm{CF}_{4}$ addition, as derived from the accordion vibration intensity gain relative to the spectral vicinity. The nozzle temperature was kept at $\approx 403 \mathrm{~K}$ with substance temperatures between 310 and $393 \mathrm{~K}$ to achieve similar mole fractions for all chain lengths. To quantify the all-trans fraction in the supersonic jet, we periodically switch between the lowfrequency range with the accordion vibration and the $\mathrm{CH}$ stretching spectral range. Comparison of the integrated scattering strength for the all-trans accordion mode with the sum of all $\mathrm{CH}$ oscillators allows for an estimate, based on corresponding B3LYP/6-311++G** predictions (see Supplement). Occasional mode mixing and Fermi resonances in the accordion range are included. ${ }^{[9]}$ Although systematic intensity errors may be in the order of $30 \%$, an estimate of the relative conformational temperature between different expansions to within $5 \mathrm{~K}$ is still possible. For this purpose, we compare to a simplified count of conformations up to 5 simultaneous gauche angles, assuming an average energy penalty of $2 \mathrm{~kJ} / \mathrm{mol}$ per gauche angle, forbidding neighboring gauche angles of opposite sign (syn-pentane effect), and including rotational symmetry numbers and enantiomeric degeneracies. $2 \mathrm{~kJ} / \mathrm{mol}$ is a compromise between higher energy single-gauche, simple double-gauche, and several lower multiple-gauche values. ${ }^{[1,5,21]}$ Figure 2 shows that the achievable all-trans fraction slowly decays from $20 \%$ at $n=13$ to less than $10 \%$ at $n=20$, being 
somewhat higher when $\mathrm{Ar}$ and in particular $\mathrm{CF}_{4}$ is added to the expansion. The drop is steeper than $1 / n$, because of contributions from populated multiple-gauche states. Applying the simple isomer count model to the experimental curves yields effective conformational temperatures of $100(\mathrm{He}), 100(\mathrm{He}+\mathrm{Ar})$, and $95 \mathrm{~K}\left(\mathrm{He}+\mathrm{CF}_{4}\right)$. Approximate fractions of single-gauche, doublegauche, and triple-gauche conformations at $100 \mathrm{~K}$ are shown. The absolute conformational temperature is less well defined. A sensitivity analysis with respect to the average gauche energy penalty and preferred isomerization of single torsional angles in the jet collisions suggests that it may be as high as $150 \mathrm{~K}$.

Most competing conformations are seen to involve one or two gauche angles in the chain, for which the low-frequency vibrations are spread over a wide spectral range. In Figure 3, they give rise to moderately structured bands in the Raman jet spectra around $200 \pm 50 \mathrm{~cm}^{-1}$ as well as to more local and weakly chain-length dependent bands near $400 \mathrm{~cm}^{-1}$. These non-specific Raman signals contrast to the sharp accordion bands at the low frequency end, to which the spectra are scaled. At nearly threefold wavenumber, one finds the next higher order accordion mode. The alltrans spectrum is simulated with dotted Gaussian line profiles $\left(4 \mathrm{~cm}^{-1} \mathrm{FWHM}\right)$ underneath the experimental spectrum. For this type of simulations, B3LYP/6-311++G** without any wavenumber-scaling has proved to be successful. ${ }^{[9]}$ With increasing chain length, the nonaccordion contribution to the spectrum grows and some bands of intermediate width start to separate from the broad background.

Spectral simulations of elementary hairpin structures are included in Fig. 3 with doubled FWHM. They involve a gauche-gauche-trans-gauche-gauche sequence in the middle of the chain with all gauche angles carrying the same sign. ${ }^{[2,22]}$ To achieve an attraction between the two parallel chain segments, it is mandatory to add a dispersion correction, for which we use Grimme's recent D3 version ${ }^{[23]}$ in combination with the B3LYP/6-311++G** method. The effect is small for an all-trans conformation but dramatic for a hairpin structure. For $\mathrm{C}_{20} \mathrm{H}_{42}$, we include the two hairpin structures with at least seven adjacent carbon atoms. The simulated hairpin abundance relative to all-trans in Fig. 3 sets an upper limit to the experimental abundance, assuming a reasonably close reproduction of the band positions and intensities. In this way, one can rule out a substantial hairpin contribution for $n=16-18$. For $n=19$, it is already close to the all-trans fraction and for $n=20$, the hairpin contribution exceeds the all-trans contribution, with experimental evidence for both shown hairpin-structures. The good wavenumber match between unscaled prediction and experiment follows the B3LYP performance for the all-trans structures. ${ }^{[9]}$ 
It suggests an outstanding stability of the employed folding motif, but we cannot rule out the presence of other suggested variants. ${ }^{[4]}$ The most prominent hairpin Raman modes fall in the range of analogous accordion modes of large cycloalkanes $\mathrm{C}_{n} \mathrm{H}_{2 n}$, which are known to prefer the same hairpin motif ${ }^{[24]}$ and to match the position of the linear alkane mode with half the number of carbon atoms. ${ }^{[25]}$

This leads to a straightforward initial assignment of $\mathrm{C}_{18} \mathrm{H}_{38}$ as the last alkane which does not prefer a folded state at low temperature, because it is the last chain which shows a negligible quantity of populated hairpin contributions in Fig. 3. However, this $n_{c}=18$ assignment may just be an upper bound. The conformational equilibrium in the jet is not complete and the all-trans structure just needs one torsional change to be populated from the abundant single-gauche structures, whereas the hairpin is handicapped by three required changes, lagging behind in population in this kinetically controlled experiment.

One could argue that $n_{c}=17$ is a more likely interpretation based on a quantum-statistical argument. While the all-trans structures and the symmetric hairpins have a rotational symmetry number of 2 due to the Pauli exclusion principle, the odd-numbered chains as well as the nonsymmetric eicosane structure share a symmetry number of 1 . This doubles their statistical weight. Furthermore, all hairpins are chiral and have an enantiomeric partner, as opposed to all-trans. Therefore, at the moment where the hairpin structures cross the energy of the all-trans structure, each of them is statistically favored by a factor of 2 or even 4 . Taking this into account, the experimental hairpin contribution for $n=17$ is less than $8 \%$ of what one would expect for the ideal isoenergetic cross-over point, whereas starting with $n=18$ (15\%) this fraction steeply increases up to $25 \%(n=19)$ and $33 \%(n=20)$. This turning point in statistical abundance could be taken as an indication that $n=18$ already starts to fold by a significant amount at $100-150 \mathrm{~K}$. Furthermore, the relaxation between structures of similar energy separated by four C-C torsion barriers is notoriously difficult in supersonic jets, ${ }^{[8]}$ possibly delaying the visible onset of folding despite its energetic feasibility.

The effective conformational temperature of $100-150 \mathrm{~K}$ involves a smearing out of the structural transition by at least $2 \mathrm{~kJ} / \mathrm{mol}$. This means that even for perfect thermal equilibrium it would be difficult to sequence conformations which are just $2 \mathrm{~kJ} / \mathrm{mol}$ apart. In summary, we can firmly state that $n_{c}=17-18$, in agreement with the quantum chemical prediction. $n_{c}=17$ is actually more likely, because even chain lengths profit somewhat more from folding than odd ones (Fig. 1). Nonadecane is definitely a hydrocarbon which prefers a folded state over a fully 
stretched one when suspended in vacuum under thermal equilibrium conditions at sufficiently low temperature. It has to be verified by experiments which achieve still lower conformational temperatures $^{[26]}$ whether octadecane may instead be named the most elementary organic foldamer. $^{[27]}$

[1] W. A. Herrebout, B. J. van der Veken, Aiying Wang, and J. R. Durig, J. Phys. Chem. 1995, 99, 578-585.

[2] Jonathan M. Goodman, J. Chem. Inf. Comput. Sci. 1997, 37, 876-878.

[3] Laura L. Thomas, Theodore J. Christakis, and William L. Jorgensen, J. Phys. Chem. B 2006, 110, 2119821204.

[4] Stefan Grimme, Jens Antony, Tobias Schwabe, and Christian Mück-Lichtenfeld, Org. Biomol. Chem. 2007, $5,741-758$.

[5] Jeffery B. Klauda, Bernard R. Brooks, Alexander D. MacKerell, Jr., Richard M. Venable, and Richard W. Pastor, J. Phys. Chem. B 2005, 109, 5300-5311.

[6] L. S. Bartell and D. A. Kohl, J. Chem. Phys. 1963, 39, 3097-3195.

[7] S. Knippenberg, Y. R. Huang, B. Hajgató, J.-P. François, J. K. Deng, and M. S. Deleuze, J. Chem. Phys. 2007, 127, 174306.

[8] Tobias N. Wassermann, Martin A. Suhm, Pascale Roubin, and Stephane Coussan, J. Mol. Struct. 2012, doi:10.1016/j.molstruc.2011.12.034.

[9] Tobias N. Wassermann, Jonas Thelemann, Philipp Zielke, and Martin A. Suhm, J. Chem. Phys. 2009, 131, 161108.

[10] R. F. Schaufele and T. Shimanouchi, J. Chem. Phys. 1967, 47, 3605-3610.

[11] C. Hampel and H. J. Werner, J. Chem. Phys. 1996, 104, 6286-6297.

[12] Hans-Joachim Werner, Frederick R. Manby, and Peter J. Knowles, J. Chem. Phys. 2003, 118, 8149-8160.

[13] Martin Schütz and Frederick R. Manby, Phys. Chem. Chem. Phys. 2003, 5, 3349-3358.

[14] Hans-Joachim Werner and Martin Schütz, J. Chem. Phys 2011, 135, 144116.

[15] Ricardo A. Mata and Hans-Joachim Werner, J. Chem. Phys. 2006, 125, 184110.

[16] Thom H. Dunning, J. Chem. Phys. 1989, 90, 1007-1023.

[17] Rick A. Kendall, Thom H. Dunning, and Robert J. Harrison, J. Chem. Phys. 1992, 96, 6796.

[18] Hans-Joachim Werner, Thomas B. Adler, and Frederick R. Manby, J. Chem. Phys. 2007, 126, 164102.

[19] Kirk A. Peterson, Thomas B. Adler, and Hans-Joachim Werner, J. Chem. Phys. 2008, 128, 084102.

[20] Tomica Hrenar, Guntram Rauhut, and Hans-Joachim Werner, J. Phys. Chem. A 2006, 110, 2060-2064.

[21] Roman M. Balabin, J. Phys. Chem. A 2009, 113, 1012-1019.

[22] Anne Zehnacker and Martin A. Suhm, Angew. Chem. Int. Ed. 2008, 47, 6970-6992; Anne Zehnacker and Martin A. Suhm, Angew. Chem. 2008, 120, 7076-7100.

[23] Stefan Grimme, Jens Antony, Stephan Ehrlich, and Helge Krieg, J. Chem. Phys. 2010, 132, 154104.

[24] J. Dale, Angew. Chem. Int. Ed. 1966, 5, 1000-1021; J. Dale, Angew. Chem. 1966, 75, 1070-1093.

[25] Hans-Peter Grossmann and Hans Bölstler, Polymer Bulletin 1981, 5, 175-177. 
[26] Samuel H. Gellman, Acc. Chem. Res. 1998, 31, 173-180.

[27] Melanie Schnell and Gerard Meijer, Angew. Chem. Int. Ed. 2009, 48, 6010-6031; Melanie Schnell and Gerard Meijer, Angew. Chem. 2009, 121, 6124-6147. 


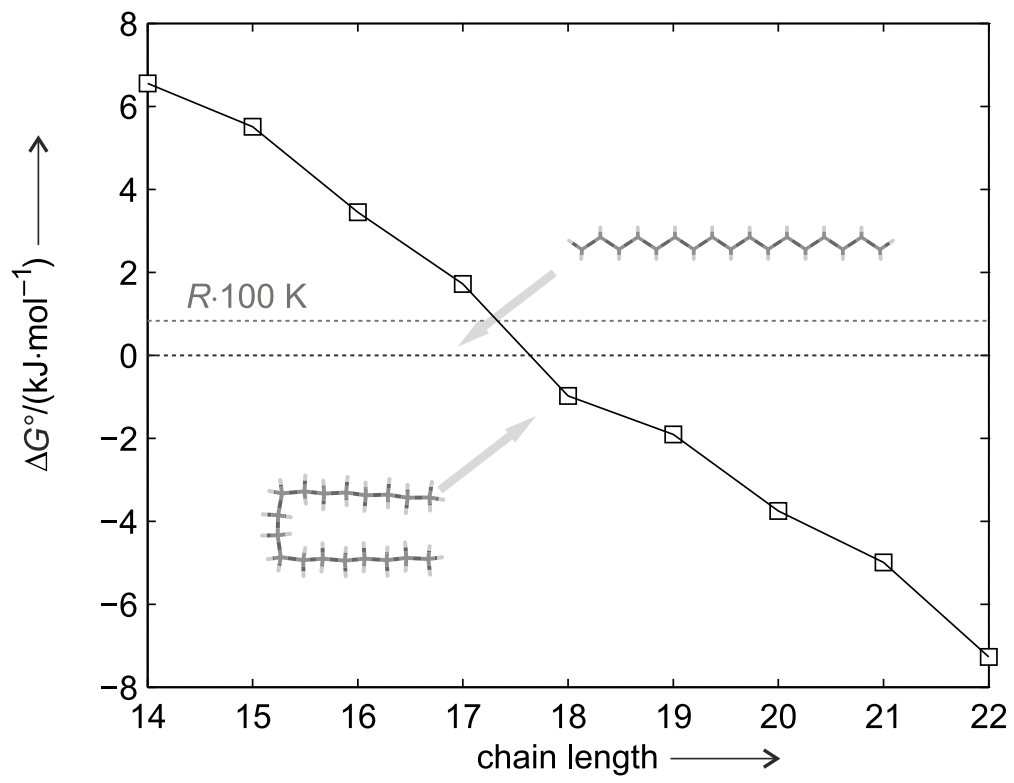

Figure 1: Calculated Gibbs energy difference $\Delta G^{\circ}=G_{\text {hairpin }}^{\circ}(100 \mathrm{~K})-G_{\text {all-trans }}^{\circ}(100 \mathrm{~K})$ vs. chain length using the energy expression given in Eq. (1), together with the scaled DF-LMP2/VTZ zero point and thermal corrections (100 K). 


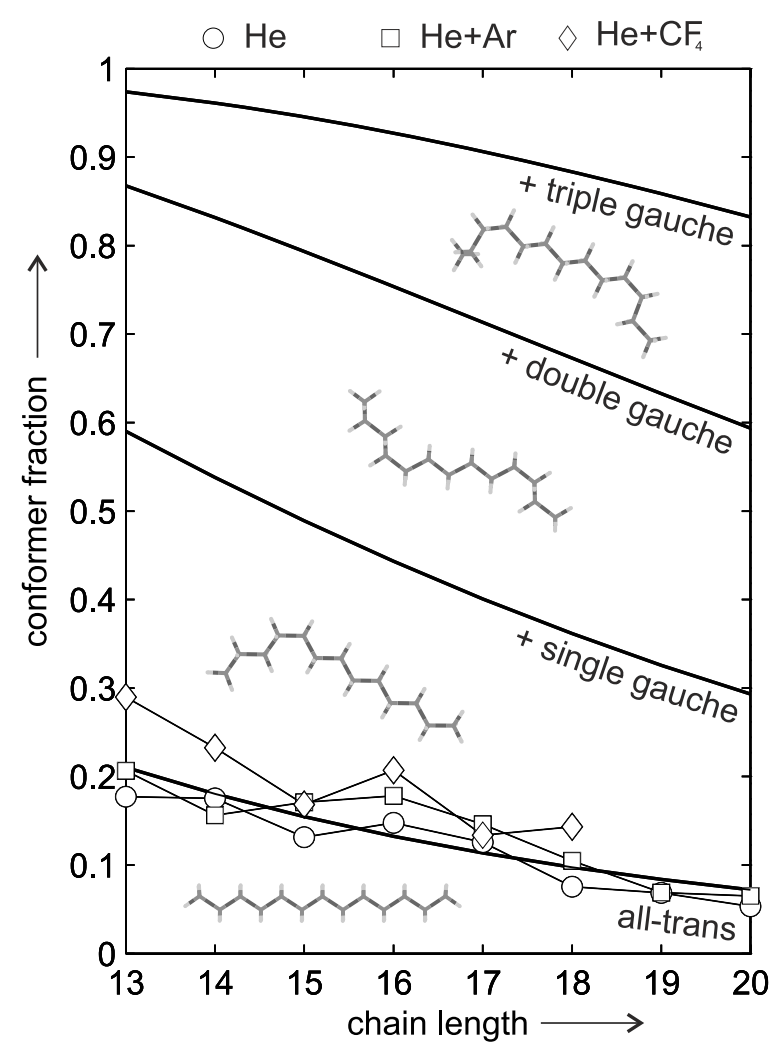

Figure 2: Cumulative abundance of no-, single-, double-, and triple-gauche conformations as a function of chain length $n$ for a conformational temperature of $100 \mathrm{~K}$. The all-trans fraction is determined semi-empirically (see Supplement) and formally corresponds to a temperature of $100 \mathrm{~K}(\mathrm{He}), 100 \mathrm{~K}(\mathrm{He}+\mathrm{Ar})$, and $95 \mathrm{~K}\left(\mathrm{He}+\mathrm{CF}_{4}\right)$. 


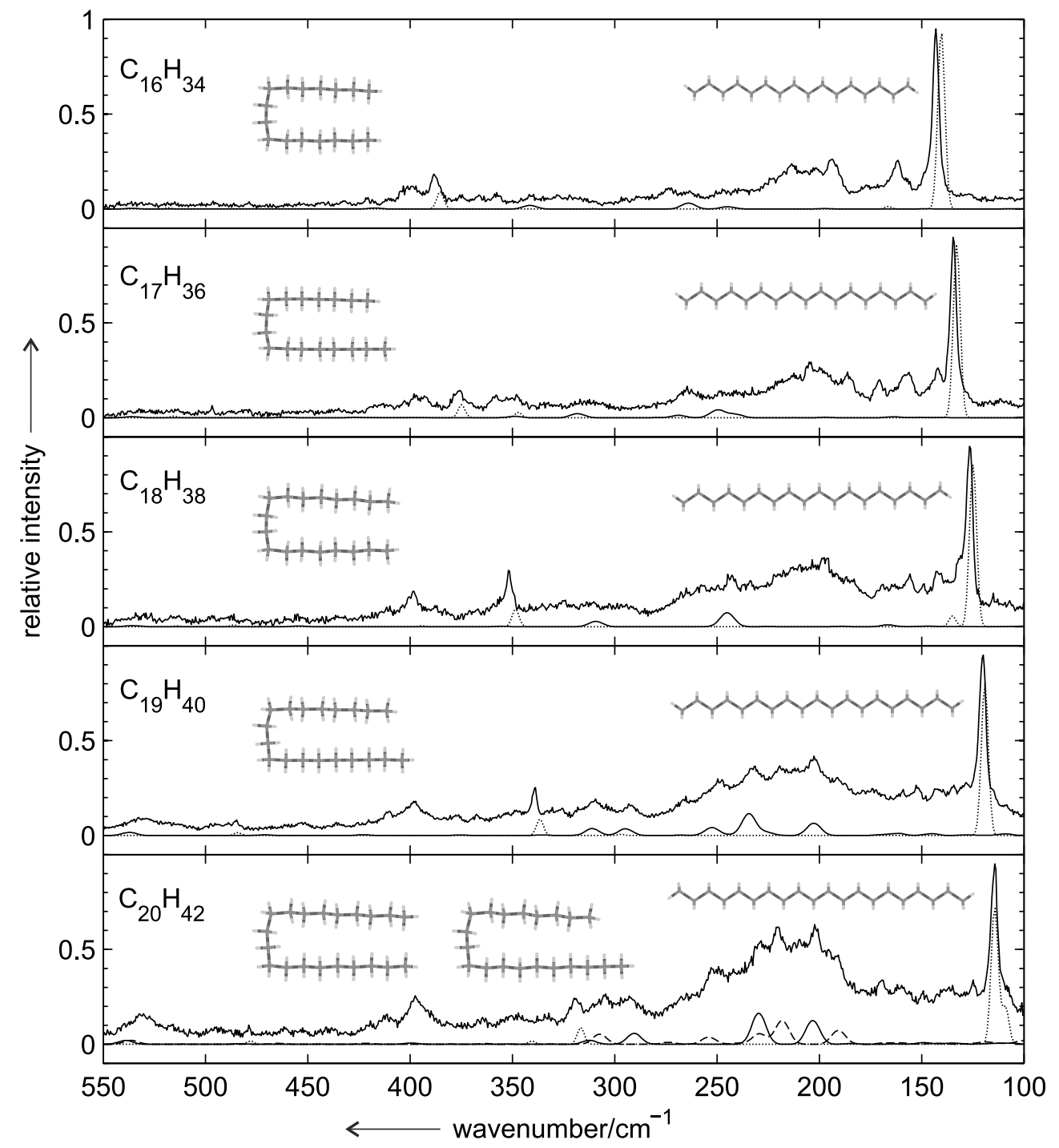

Figure 3: Experimental and simulated low frequency Raman jet spectra in He expansions of hexadecane to eicosane. The all-trans spectrum is shown with dotted lines, upper bounds of the hairpin conformer are shown with solid and dashed lines (nonsymmetric eicosane). The visually estimated abundance ratios for the scaling of the simulated spectra are (hairpin:all-trans) $0.2: 1$, 0.3:1, 0.3:1, 1:1, and (1+1):1 for hexa-, hepta-, octa-, nonadecane, and eicosane, respectively. 


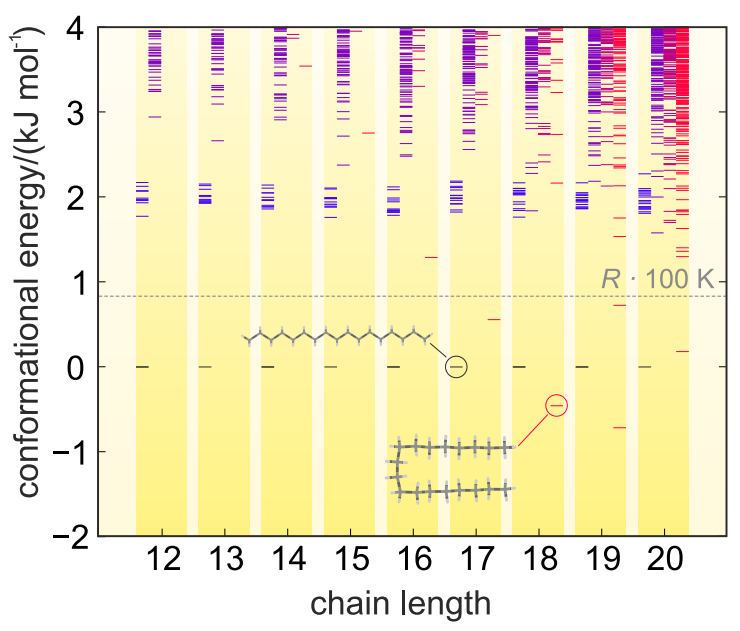

TOC image 